\title{
Applied Sumudu Transform with Adomian Decomposition Method to the Coupled Drinfeld-Sokolov-Wilson System
}

\author{
Abdulghafor M.A. Al-Rozbayani \\ abdulghafor_rozbayani@uomosul.edu.iq \\ Department of Mathematics, \\ College of Computer Science and Mathematics \\ University of Mosul, IRAQ
}

\author{
Ammar H. Ali \\ ammar.csp108@student.uomosul.edu.iq \\ Directorate of Education in Nineveh, \\ Mosul, IRAQ
}

Received on: 04/02/2021

Accepted on: 04/04/2021

\begin{abstract}
In this paper, we studied and applied a modern numerical method, which is combining Sumudu transform with Adomian decomposition Method to obtain approximate solutions of the nonlinear the Coupled Drinfeld-Sokolov-Wilson (DSW) system. Positive and negative values of the variable $\mathrm{x}$ and various values of the variable $\mathrm{t}$ were taken with the initial conditions of the system as well as the values of the parameters $(\alpha, \beta, \gamma, \varepsilon$ and $\mathrm{c})$. The efficiency of the method was verified, as the results obtained were compared with the accurate solution of the system. We noticed that the results are very accurate and the effectiveness of the method was confirmed.
\end{abstract}

Keywords: Sumudu transform, Adomian decomposition method, Drinfeld-SokolovWilson.

\section{Introduction}

Drinfeld-Sokolov-Wilson (DSW) system [6, 7, and 19] has many uses in solving nonlinear partial differential equations, as this system provides a set of convergent numerical solutions to its users. That the first to discover this system were Drinfeld and Sokolov [5, 6] and Wilson [17], and that this system depends on two basic parameters and contains random constants that are non-zero. The Drinfeld-Sokolov-Wilson (DSW) system was solved using several numerical methods including the Homotopy analysis method [1, 11, and 13], the F-expansion method [18], the decomposition method [8], the Tan method [10] and many more methods. In this paper, we will use the Sumudu transformation with Adomian decomposition method to solve this system in a numerical analysis method.

Sumudu transform with Adomian decomposition method was used by the researchers (Trushit Patel and Ramakanta Meher) [12] in their research (Adomian Decomposition Sumudu Transform Method for Solving Fully Nonlinear Fractional Order Power-Law Fin-Type Problems), widely acceptable results were obtained, which indicates the possibility of using Sumudu transform with Adomian decomposition method to solve many non-linear problems.

In the first section of this paper, an introduction to the (DSW) system and its uses was presented, as well as an introduction to Sumudu transform with Adomian decomposition method. In the second section, the mathematical model of the system (DSW) is presented. In the third section it was shown Describe Sumudu Transform with Adomian Decomposition Method to the Coupled Drinfeld - Sokolov - Wilson (DSW) System. In the fourth section Applied Sumudu transform with Adomian decomposition method to the coupled Drinfeld-Sokolov-Wilson system. In the fifth section, we 
presented the numerical results represented by the absolute error value in the form of tables and graphs. Finally, in the sixth section, conclusions are presented.

\section{Mathematical model}

Considering that form the Coupled Drinfeld-Sokolov-Wilson (DSW) system as the formula:

$u_{t}+\alpha v v_{x}=0$

$v_{t}+\beta v_{x x x}+\gamma u v_{x}+\varepsilon u_{x} v=0$

Where $(\alpha, \beta, \gamma, \varepsilon)$ are nonzero parameters, time $(\mathrm{t})$, space $(\mathrm{x})$ are the independent variables and $u(x, t), v(x, t)$ are the dependent variables.

Where $\alpha=3, \beta=\gamma=2$ and $\varepsilon=1$, depending on the initial values [19]

$$
\begin{aligned}
& u(x, 0)=\frac{6 c}{(\gamma+2 \varepsilon)}\left(\operatorname{sech}\left(\sqrt{\frac{c}{\beta}}(x)\right)\right) \\
& v(x, 0)=2 \sqrt{3} \sqrt{\frac{c^{2}}{\alpha(\gamma+2 \varepsilon)}}\left(\operatorname{sech}\left(\sqrt{\frac{c}{\beta}}(x)\right)\right)
\end{aligned}
$$

And that the exact solution to the system (1) is

$$
\begin{aligned}
& u(x, t)=\frac{6 c}{(\gamma+2 \varepsilon)}\left(\operatorname{sech}\left(\sqrt{\frac{c}{\beta}}(x-c t)\right)\right)^{2} \\
& v(x, t)=2 \sqrt{3} \sqrt{\frac{c^{2}}{\alpha(\gamma+2 \varepsilon)}}\left(\operatorname{sech}\left(\sqrt{\frac{c}{\beta}}(x-c t)\right)\right)
\end{aligned}
$$

\section{Describe Sumudu Transform with Adomian Decomposition Method to the Coupled Drinfeld-Sokolov-Wilson (DSW) System}

Sumudu transform can address many problems in engineering mathematics and applied science, the conversion of Sumudu transform was originally proposed by Watugala $[14,15]$ to solve differential equations and control engineering problems.

The Sumudu transform is defined of the function $f(t)$ :

$$
\begin{aligned}
& A=\left\{f(t)\left|\exists M, T_{1}, T_{2}>0,\right| f(t) \mid<M e^{\frac{|t|}{T_{j}}}, \text { if } t \in(-1)^{j} \times[0, \infty]\right\}, \text { By } \\
& G(u)=S[f(t)]=\int_{0}^{\infty} f(u t) e^{-t} d t, u \in\left(-T_{1}, T_{2}\right), \text { or } \\
& G(u)=S[f(t)]=\frac{1}{u} \int_{0}^{\infty} f(t) e^{\frac{-t}{u}} d t, u \in\left(-T_{1}, T_{2}\right) .
\end{aligned}
$$

Where $S$ is sumudu transform operator

While on the other hand let the variable $t$ inverse Sumudu transform to variable $w$. Therefore Discrete

Inverse Sumudu transform of $f(t)$ in $A$ is [3] ,

$S^{-1}[f(t)]=F_{-1}(w)=\sum_{n=0}^{\infty} \frac{f^{(n)}(0) w^{n}}{(n !)^{2}}$.

The Sumudu transform was used on linear equations $\left(u_{t}, v_{t}\right.$ and $\left.v_{x x x}\right)[2,4$, and 9]

$$
\begin{aligned}
& S\left[u_{t}\right]=\frac{1}{w}[U(x, w)-u(x, 0)] \\
& S\left[v_{t}\right]=\frac{1}{w}[V(x, w)-v(x, 0)] \\
& S\left[v_{x x x}\right]=\frac{d^{3} V(x, w)}{d x^{3}}
\end{aligned}
$$

When $U(x, w)$ is sumudu transform of $u(x, t)$ and $V(x, w)$ is sumudu transform of $v(x, t)$

We also used Adomian decomposition method on nonlinear equations. 
Adomian Decomposition Method It can be used and applied easily in many linear problems and equations in various sciences and mathematical, Adomian decomposition method in its modified form was introduced by Wazwaz [16], this method provides accelerated approximate solutions, and it can be combined with Sumudu transform to solve some problems related to nonlinear partial differential equations.

If $F(u)=u u_{x}$ yields,

$F(u)=\left(u_{0}+u_{1}+u_{2}+u_{3}+\cdots\right) *\left(u_{0 x}+u_{1 x}+u_{2 x}+u_{3 x}+\cdots\right)$

Multiplying the two factors gives

$$
\begin{aligned}
& F(u)=u_{0 x} u_{0}+u_{0 x} u_{1}+u_{1 x} u_{0}+u_{0 x} u_{2}+u_{1 x} u_{1}+u_{2 x} u_{0}+u_{0 x} u_{3}+u_{1 x} u_{2}+ \\
& u_{2 x} u_{1}+u_{3 x} u_{0}+\cdots \\
& \text { It then follows that Adomian polynomials are given by } \\
& A_{0}=u_{0 x} u_{0} \text {, } \\
& A_{1}=u_{0 x} u_{1}+u_{1 x} u_{0} \text {, } \\
& A_{2}=u_{0 x} u_{2}+u_{1 x} u_{1}+u_{2 x} u_{0} \text {, } \\
& A_{3}=u_{0 x} u_{3}+u_{1 x} u_{2}+u_{2 x} u_{1}+u_{3 x} u_{0} \text {, }
\end{aligned}
$$

\section{Application}

Taking Sumudu transform of both sides equations (1)

$S\left[u_{t}\right]+\alpha S\left[v v_{x}\right]=0$,

$S\left[v_{t}\right]+\beta S\left[v_{x x x}\right]+\gamma S\left[u v_{x}\right]+\varepsilon S\left[u_{x} v\right]=0$

Substituting equations $(7,8)$ and equations $(9)$ in to equations (10) gives:

$$
\begin{aligned}
& \frac{1}{w}[U(x, w)-u(x, 0)]+\alpha S\left[v v_{x}\right]=0, \\
& \frac{1}{w}[V(x, w)-v(x, 0)]+\beta\left(\frac{\partial^{3} V(x, w)}{\partial x^{3}}\right)+\gamma S\left[u v_{x}\right]+\varepsilon S\left[u_{x} v\right]=0 \\
& U(x, w)=u(x, 0)-w \alpha S\left[v v_{x}\right], \\
& V(x, w)=v(x, 0)-w \beta\left(\frac{\partial^{3} V(x, w)}{\partial x^{3}}\right)-w \gamma S\left[u v_{x}\right]-w \varepsilon S\left[u_{x} v\right]
\end{aligned}
$$

Substituting equations $(2,3)$ in to equations (12):

$$
\begin{aligned}
& U(x, w)=\frac{6 c}{(\gamma+2 \varepsilon)}\left(\operatorname{sech}\left[\sqrt{\frac{c}{\beta}}(x)\right]\right)-w \alpha S\left[v v_{x}\right], \\
& V(x, w)=2 \sqrt{3} \sqrt{\frac{c^{2}}{\alpha(\gamma+2 \varepsilon)}}\left(\operatorname{sech}\left(\sqrt{\frac{c}{\beta}}(x)\right)\right)-w \beta\left(\frac{\partial^{3} S[v]}{\partial x^{3}}\right)-w \gamma S\left[u v_{x}\right]-w \varepsilon S\left[u_{x} v\right]
\end{aligned}
$$

Taking the inverse Sumudu transform:

$$
\begin{aligned}
& u=\frac{6 c}{\gamma+2 \varepsilon} \operatorname{sech}\left(\sqrt{\frac{c}{\beta}} x\right)-S^{-1}\left\{w \alpha S\left[v v_{x}\right]\right\}, \\
& \left.v=2 \sqrt{3} \sqrt{\frac{c^{2}}{\alpha(\gamma+2 \varepsilon)}}\right)\left(\operatorname{sech}\left[\sqrt{\frac{c}{\beta}}(x)\right]\right)-S^{-1}\left\{w \beta \frac{\partial^{3} S[v]}{\partial x^{3}}+w \gamma S\left[u v_{x}\right]+w \varepsilon S\left[u_{x} v\right]\right\}
\end{aligned}
$$

The solutions, $v$ can be written as

$$
\begin{aligned}
& u(x, t)=\sum_{n=0}^{\infty} u_{n}(x, t), \\
& v(x, t)=\sum_{n=0}^{\infty} v_{n}(x, t)
\end{aligned}
$$

And the nonlinear terms

$$
\begin{aligned}
& v v_{x}=\sum_{n=0}^{\infty} A_{n}, \\
& u v_{x}=\sum_{n=0}^{\infty} B_{n}, \\
& u_{x} v=\sum_{n=0}^{\infty} C_{n}
\end{aligned}
$$

Substituting equations (15) and (16) in to equations (14)

$\sum_{n=0}^{\infty} u_{n}=\frac{6 c}{\gamma+2 \varepsilon} \operatorname{sech}\left(\sqrt{\frac{c}{\beta}} x\right)-S^{-1}\left\{w \alpha S\left[\sum_{n=0}^{\infty} A_{n}\right]\right\}$, 
$\left.\sum_{n=0}^{\infty} v_{n}=2 \sqrt{3} \sqrt{\frac{c^{2}}{\alpha(\gamma+2 \varepsilon)}}\right)\left(\operatorname{sech}\left[\sqrt{\frac{c}{\beta}}(x)\right]\right)-S^{-1}\left\{w \beta \frac{\partial^{3} S\left[\sum_{n=0}^{\infty} v_{n}\right]}{\partial x^{3}}+w \gamma S\left[\sum_{n=0}^{\infty} B_{n}\right]+\right.$ $\left.w \varepsilon S\left[\sum_{n=0}^{\infty} C_{n}\right]\right\}$

The recursive relations are given by

$u_{n+1}=-S^{-1}\left\{w \alpha S\left[A_{n}\right]\right\}$,

$v_{n+1}=-S^{-1}\left\{w \beta \frac{\partial^{3} S\left[v_{n}\right]}{\partial x^{3}}+w \gamma S\left[B_{n}\right]+w \varepsilon S\left[C_{n}\right]\right\},(n=0,1,2, \ldots)$

Where

$$
\begin{aligned}
& u_{o}=\frac{6 c}{(\gamma+2 \varepsilon)}\left(\operatorname{sech}\left(\sqrt{\frac{c}{\beta}}(x)\right)\right), \\
& v_{o}=2 \sqrt{3} \sqrt{\frac{c^{2}}{\alpha(\gamma+2 \varepsilon)}}\left(\operatorname{sech}\left(\sqrt{\frac{c}{\beta}}(x)\right)\right)
\end{aligned}
$$

The first few components of nonlinear terms are

$A_{0}=v_{0} v_{0 x}$

$A_{1}=v_{0} v_{1 x}+v_{1} v_{0 x}$

$A_{2}=v_{0} v_{2 x}+v_{1} v_{1 x}+v_{2} v_{0 x}$

$B_{0}=u_{0} v_{0 x}$

$B_{1}=u_{0} v_{1 x}+u_{1} v_{0 x}$

$B_{2}=u_{0} v_{2 x}+u_{1} v_{1 x}+u_{2} v_{0 x}$

$C_{0}=u_{0 x} v_{0}$

$C_{1}=u_{0 x} v_{1}+u_{1 x} v_{0}$

$C_{2}=u_{0 x} v_{2}+u_{1 x} v_{1}+u_{2 x} v_{0}$

Applying the above equations, we get

$u_{1}=\frac{12 t c^{2} \sqrt{\frac{c}{\beta}} \sinh \left(\sqrt{\frac{c}{\beta}} x\right)}{(\gamma+2 \varepsilon) \cosh \left(\sqrt{\frac{c}{\beta}} x\right)^{3}}$

$v_{1}=\frac{1}{\cosh \left(\sqrt{\frac{c}{\beta}} x\right)^{4}(\gamma+2 \varepsilon)}\left(2 t \sinh \left(\sqrt{\frac{c}{\beta}} x\right) \sqrt{3} \sqrt{\frac{c^{2}}{\alpha(\gamma+2 \varepsilon)}} \sqrt{\frac{c}{\beta}} c\right.$

$\left(\cosh \left(\sqrt{\frac{c}{\beta}} x\right)^{2} \gamma+2 \cosh \left(\sqrt{\frac{c}{\beta}} x\right)^{2} \varepsilon-6 \gamma-12 \varepsilon+6 \gamma \cosh \left(\sqrt{\frac{c}{\beta}} x\right)+\right.$

$\left.\left.6 \varepsilon \cosh \left(\sqrt{\frac{c}{\beta}} x\right)\right)\right)$

$u_{2}=$

$\frac{1}{\beta(\gamma+2 \varepsilon)^{2} \cosh \left(\sqrt{\frac{c}{\beta}} x\right)^{6}}\left(6 t^{2} c^{4}\left(18 \gamma \cosh \left(\sqrt{\frac{c}{\beta}} x\right)^{3}-24 \gamma \cosh \left(\sqrt{\frac{c}{\beta}} x\right)-27 \cosh \left(\sqrt{\frac{c}{\beta}} x\right)^{2} \gamma+\right.\right.$ $2 \gamma \cosh \left(\sqrt{\frac{c}{\beta}} x\right)^{4}+30 \gamma+4 \cosh \left(\sqrt{\frac{c}{\beta}} x\right)^{4} \varepsilon-54 \cosh \left(\sqrt{\frac{c}{\beta}} x\right)^{2} \varepsilon+18 \cosh \left(\sqrt{\frac{c}{\beta}} x\right)^{3} \epsilon-$ $\left.\left.24 \varepsilon \cosh \left(\sqrt{\frac{c}{\beta}} x\right)+60 \varepsilon\right)\right)$

$v_{2}=\frac{1}{\beta(\gamma+2 \varepsilon)^{2} \cosh \left(\sqrt{\frac{c}{\beta}} x\right)^{7}}\left(t^{2} c^{3} \sqrt{\frac{c^{2}}{\alpha(\gamma+2 \varepsilon)}} \sqrt{3}\left(-720 \gamma^{2}-2880 \gamma \varepsilon-2880 \varepsilon^{2}+\right.\right.$

$3156 \cosh \left(\sqrt{\frac{c}{\beta}} x\right)^{2} \gamma \varepsilon+1404 \gamma \cosh \left(\sqrt{\frac{c}{\beta}} x\right) \varepsilon-1362 \cosh \left(\sqrt{\frac{c}{\beta}} x\right)^{3} \varepsilon \gamma-$

$572 \cosh \left(\sqrt{\frac{c}{\beta}} x\right)^{4} \varepsilon \gamma+4 \cosh \left(\sqrt{\frac{c}{\beta}} x\right)^{6} \gamma \varepsilon+162 \cosh \left(\sqrt{\frac{c}{\beta}} x\right)^{5} \varepsilon \gamma+$ 


$$
\begin{aligned}
& 720 \cosh \left(\sqrt{\frac{c}{\beta}} x\right)^{2} \gamma^{2}+3252 \cosh \left(\sqrt{\frac{c}{\beta}} x\right)^{2} \varepsilon^{2}+504 \gamma^{2} \cosh \left(\sqrt{\frac{c}{\beta}} x\right)+ \\
& 792 \varepsilon^{2} \cosh \left(\sqrt{\frac{c}{\beta}} x\right)-804 \cosh \left(\sqrt{\frac{c}{\beta}} x\right)^{3} \varepsilon^{2}-644 \cosh \left(\sqrt{\frac{c}{\beta}} x\right)^{4} \varepsilon^{2}- \\
& 480 \cosh \left(\sqrt{\frac{c}{\beta}} x\right)^{3} \gamma^{2}-98 \cosh \left(\sqrt{\frac{c}{\beta}} x\right)^{4} \gamma^{2}-\cosh \left(\sqrt{\frac{c}{\beta}} x\right)^{6} \gamma^{2}+4 \cosh \left(\sqrt{\frac{c}{\beta}} x\right)^{6} \varepsilon^{2}+ \\
& \left.\left.54 \cosh \left(\sqrt{\frac{c}{\beta}} x\right)^{5} \gamma^{2}+108 \cosh \left(\sqrt{\frac{c}{\beta}} x\right)^{5} \varepsilon^{2}\right)\right)
\end{aligned}
$$

So the approximate solution

$$
\begin{aligned}
& U_{a p p}(x, t)=\sum_{n=0}^{3} u_{n} \\
& V_{a p p}(x, t)=\sum_{n=0}^{3} v_{n}
\end{aligned}
$$

The equation (20), (21) is a numerical solution for (DSW) system by using Sumudu transform with Adomian decomposition method.

Maple codes are used to solve these equations

\section{Numerical results}

In this part, some tables and drawings were included, where positive and negative values of the variable $X$ were taken and different values were imposed for the variable $t$ and they were computed in the original equation and in the approximate equation, in table 1 the value of the absolute error between $u$ approximation and $u$ exact, table 2 the value of the absolute error between $v$ approximation and v exact, Figure 1, we show $u$ approximation, $\mathrm{u}$ exact, $\mathrm{v}$ approximation and $\mathrm{v}$ exact in graphically. Figure 2, we show absolute error of $\mathrm{u}$ approximation, $\mathrm{u}$ exact and absolute error of $\mathrm{v}$ approximation, $\mathrm{v}$ exact in a graphically.

\section{Table 1:}

The absolute error between $u_{\text {exact }}$ and $u_{\text {app }}$,

$$
U_{\text {Error }}=\left|u_{\text {exact }}-u_{\text {app }}\right|
$$

By using Sumudu transform with Adomian decomposition Method,

$$
\begin{aligned}
& \text { When } \quad \alpha=3, \beta=\gamma=2 \text { and } \varepsilon=1 \text {, } \\
& x=\{-6,-4,-2,0,2,4,6\}, t=\{0.1,0.2,0.3,0.4,0.5,0.6\} \text { and } c=0.1
\end{aligned}
$$

\begin{tabular}{|c|l|l|l|l|l|l|}
\hline$x / t$ & \multicolumn{1}{|c|}{0.1} & \multicolumn{1}{|c|}{0.2} & \multicolumn{1}{|c|}{0.3} & \multicolumn{1}{|c|}{0.4} & \multicolumn{1}{c|}{0.5} & \multicolumn{1}{c|}{0.6} \\
\hline-6 & 0.03748319561 & 0.03748345758 & 0.03748390345 & 0.03748454075 & 0.03748537709 & 0.03748642002 \\
\hline-4 & 0.03146463818 & 0.03146309603 & 0.03146039558 & 0.03145643088 & 0.03145109533 & 0.03144428290 \\
\hline-2 & 0.0125659713 & 0.0125650238 & 0.0125636491 & 0.0125620146 & 0.0125602877 & 0.0125586357 \\
\hline 0 & 0.0000011250 & 0.0000045000 & 0.0000101248 & 0.0000179994 & 0.0000281234 & 0.0000404967 \\
\hline 2 & 0.0125659155 & 0.0125645775 & 0.0125621432 & 0.0125584449 & 0.0125533155 & 0.0125465877 \\
\hline 4 & 0.03146467366 & 0.03146337964 & 0.03146135322 & 0.03145870048 & 0.03145552822 & 0.03145194288 \\
\hline 6 & 0.03748319308 & 0.03748343744 & 0.03748383545 & 0.03748437958 & 0.03748506233 & 0.03748587614 \\
\hline
\end{tabular}

\section{Table 2:}

The absolute error between $v_{\text {exact }}$ and $v_{\text {app }}, V_{\text {Error }}=\left|v_{\text {exact }}-v_{\text {app }}\right|$. By using Sumudu transform with Adomian decomposition Method,

When $\alpha=3, \beta=\gamma=2$ and $\varepsilon=1$, $x=\{-6,-4,-2,0,2,4,6\}, t=\{0.1,0.2,0.3,0.4,0.5,0.6\}$ and $c=0.1$ 


\begin{tabular}{|c|c|c|c|c|c|c|}
\hline$x / t$ & 0.1 & 0.2 & 0.3 & 0.4 & 0.5 & 0.6 \\
\hline-6 & 0.00007402050 & 0.00015024613 & 0.00022900558 & 0.00031062755 & 0.00039544076 & 0.00048377393 \\
\hline-4 & 0.00002189038 & 0.00004060372 & 0.00005536804 & 0.00006541112 & 0.00006996088 & 0.00006824516 \\
\hline-2 & 0.00007603121 & 0.00015750387 & 0.00024393850 & 0.00033485557 & 0.00042977555 & 0.00052821893 \\
\hline 0 & 0.00000637503 & 0.00002550006 & 0.00005737509 & 0.00010200014 & 0.00015937538 & 0.00022950069 \\
\hline 2 & 0.00007011015 & 0.00013381987 & 0.00019064956 & 0.00024011968 & 0.00028175082 & 0.00031506345 \\
\hline 4 & 0.00002429531 & 0.00005022341 & 0.00007701223 & 0.00010388974 & 0.00013008385 & 0.00015482244 \\
\hline 6 & 0.00007214412 & 0.00014274057 & 0.00021211807 & 0.00028060536 & 0.00034853116 & 0.00041622419 \\
\hline
\end{tabular}

(a) Uapp

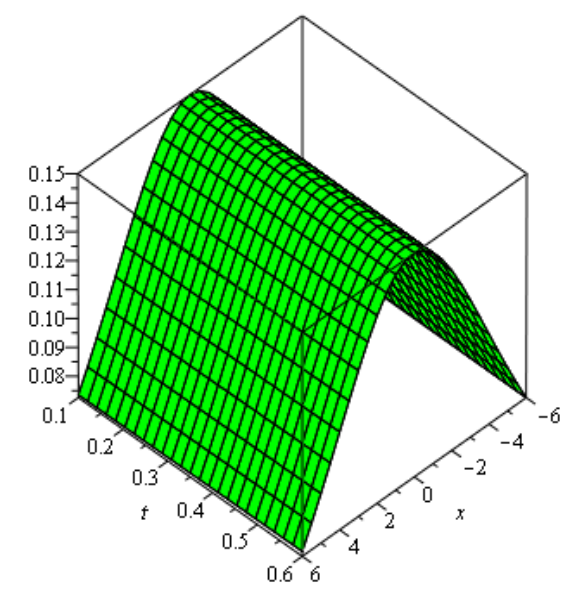

(c)

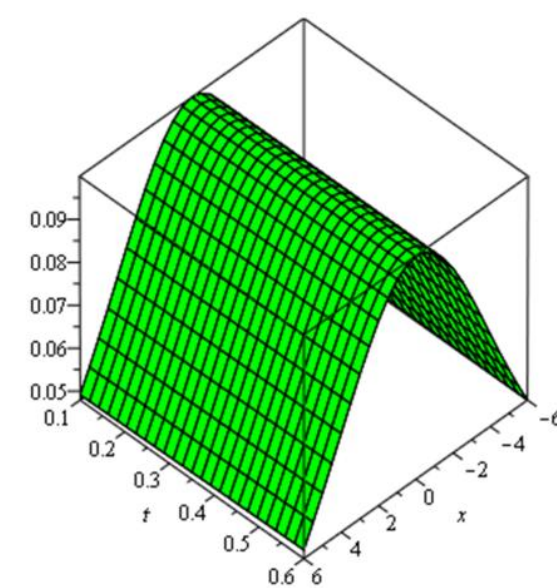

(b) Uexact

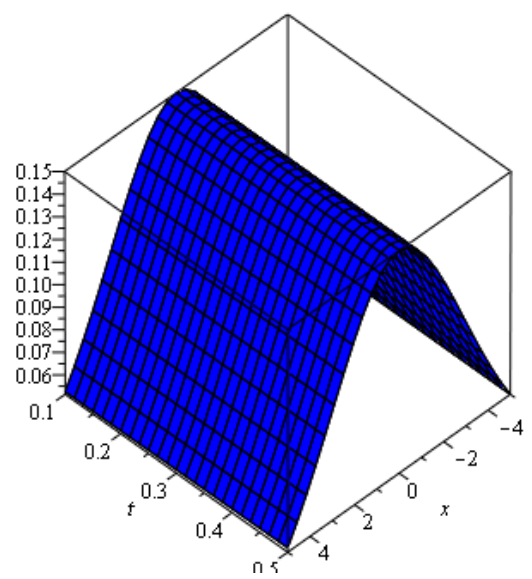

(d) exact

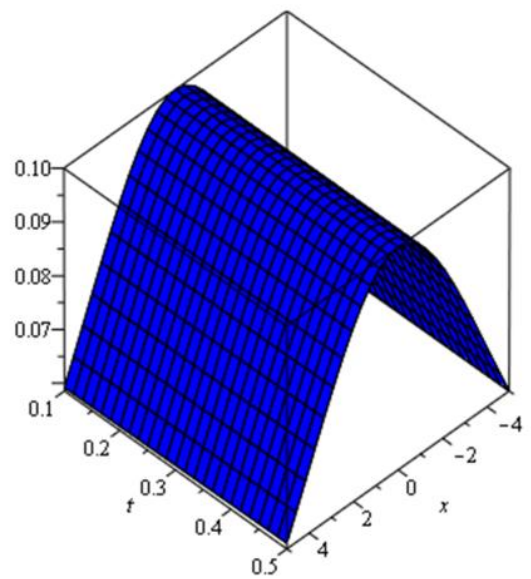

\section{Figure 1:}

(a) $u(x, t)$ Approximate $\left(U_{a p p}\right)$

(b) $u(x, t)$ Exact $\left(U_{\text {exact }}\right)$

(c) $v(x, t)$ Approximate $\left(V_{a p p}\right)$

(d) $v(x, t)$ Exact $\left(V_{\text {exact }}\right)$

By using Sumudu transform with Adomian decomposition Method,

When $\alpha=3, \beta=\gamma=2$ and $\varepsilon=1$,

$x=\{-6,-4,-2,0,2,4,6\}, t=\{0.1,0.2,0.3,0.4,0.5,0.6\}$ and $c=0.1$

(a)

(b) 

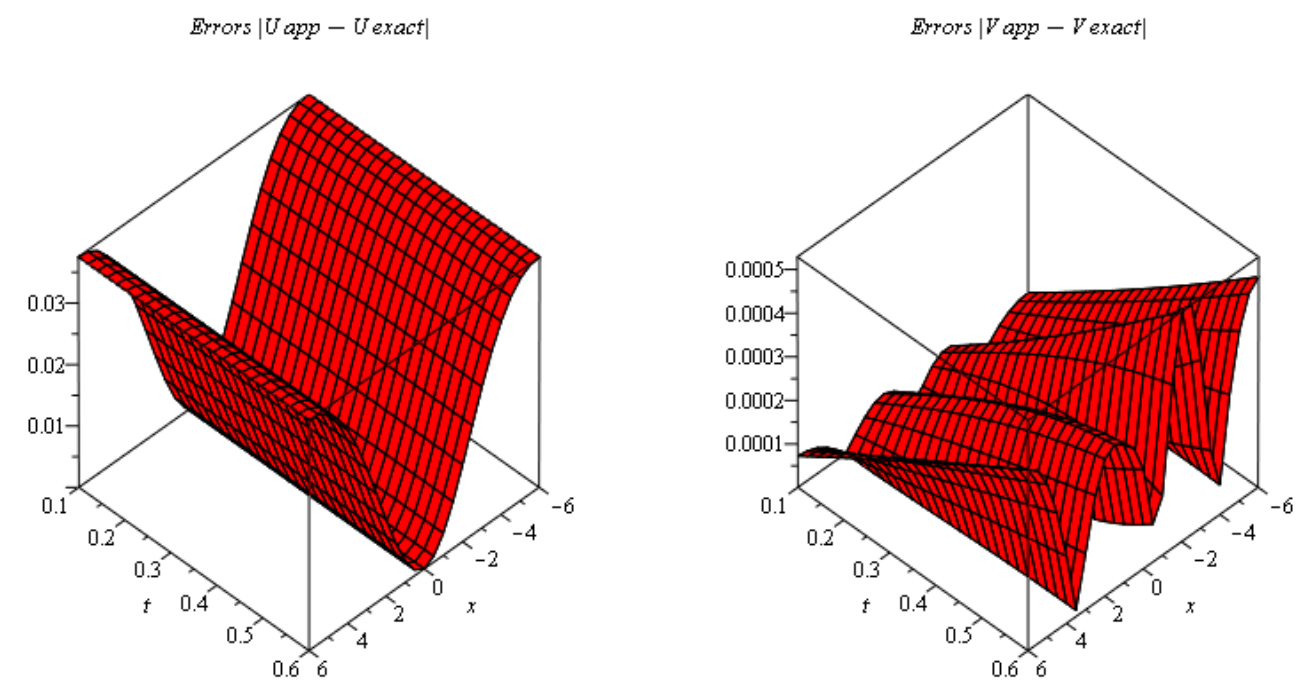

Figure 2:

(a) The absolute error between $u_{\text {exact }}$ and $u_{\text {app }}, U_{\text {Error }}=\left|u_{\text {app }}-u_{\text {exact }}\right|$

(b) The absolute error between $v_{\text {exact }}$ and $v_{\text {app }}, V_{\text {Error }}=\left|v_{a p p}-v_{\text {exact }}\right|$

Using Sumudu transform with Adomian decomposition Method,

When $\alpha=3, \beta=\gamma=2$ and $\varepsilon=1$,

$x=\{-6,-4,-2,0,2,4,6\}, t=\{0.1,0.2,0.3,0.4,0.5,0.6\}$ and $c=0.1$

\section{Conclusion}

In this paper, a new method was applied to solve the Coupled Drinfeld-SokolovWilson system where the Sumudu transform method was combined With Adomian decomposition method to solve this system, and different values of the variables were relied upon, and it was concluded that this method is very strong and effective because it gives numerical solutions very close to the exact solution of this system. 


\section{REFERENCES}

[1] Arora , R.and kumar, A." Solution of the Coupled Drinfeld-Sokolov-Wilson (DSW) System by Homotopy Analysis Method", Advanced Science, Engineering and Medicine Vol. 5, pp. 1-7, (2013)

[2] Belgacem F. B. M. And Karaballi A. A., "Sumudu transform fundament Properties investigations and applications," Journal Applied Mathematics and Stochastic Analysis, vol. 2006, Article ID 91083, 23 pages, 2006.

[3] Belgacem F.B.M. and Silambarasan R., A distinctive Sumudu treatment of trigonometric functions. Journal. Of Compute. Appl. Math. 312, pp. 74-81. 2017.

[4] Bulut H., Baskonus H. M., and Belgacem F. B. M., "The analytical solution of some fractional ordinary differential equations by the Sumudu transform method," Abstract and Applied Analysis, vol. 2013, Article ID 203875, 6 pages, 2013.

[5] Drinfeld, V. G. and Sokolov, V. V. Lie algebras and equations of Korteweg-de Vries type, J. Sov. Math. 30, 1975-2005(1985).

[6] Drinfeld, V. G. and Sokolov, V. V., Equations of Korteweg-de Vries type and simple Lie algebras, Sov. Math. Dokl. 23, 457-462(1981).

[7] Haq, S. , Hassan, N. , Tirmizi, S. I. A. , and Usman, M. , A Meshless Method of Lines for the Numerical Solution of Coupled Drinfeld-Sokolov-Wilson System, Preprint submitted to Elsevier (2010).

[8] Inc, M. On numerical doubly periodic wave solutions of the coupled DrinfeldSokolov-Wilson equation by the decomposition method, Applied Mathematics and Computation, 172(1), 421-430(2006).

[9] Katatbeh Q. D. and Belgacem F. B. M., "Applications of the Sumudu transformto fractional differential equations," Nonlinear Studies, vol. 18, no. 1, pp. 99-112, 2011.

[10] Li, Z. B. Traveling wave solutions of nonlinear mathematical physics equations, Science Press (Beijing), 2007.

[11] Nadeem, S., Hussain, A. , and Khan, M. , Commun. Nonlinear Sci. Numer. Simul. 15, 481 (2010).

[12] Patel T. and Meher R. " Adomian Decomposition Sumudu Transform Method for Solving Fully Nonlinear Fractional Order Power- Law Fin-Type Problems " Vol. 27; Issue No. 2; Year 2016.

[13] Shendkar, A.M. and Jadhav, P.V., Elzaki Transform: ASolution of Differential Equations, International Journal of Science,Engineering and Technology Research, 4(4), pp. 1006-1008, 2015.

[14] Watugala, G. K. 1998. Sumudu transform a new integral transform to solve differential equations and control engineering problems. Mathematical Engineering in Industry. 6(4): 319-329.

[15] Watugala, G. K. 2002. The Sumudu transform for functions of two variables. Mathematical Engineering in Industry. 8(4), 293-302 
[16] Wazwaz, A.M. Partial Differential Equations and Solitary Waves Theory, Higher Education Press, Beijing, 2009, Springer-Verlag, Berlin.

[17] Wilson, G. The affine Lie algebra and an equation of Hirota and Satsuma, Phys.Lett. A, 89(7), 332-334(1982).

[18] Zha, X. Q. and Zhi, H. Y. An Improved F.Expansion Method and Its Application to Coupled Drinfeld-Sokolov-W ilson Equation, Commun.Theor.Phys, 50, 309-314(2008).

[19] Zhang, W.-M., Applied Mathematical Sciences 38, 1894 (2011). 